\title{
15
}

\section{QUORUM-QUENCHING ACYLASES IN PSEUDOMONAS AERUGINOSA}

\author{
Joanna Krzeslak, Mariana Wahjudi, and Wim J. Quax* \\ Department of Pharmaceutical Biology \\ University of Groningen \\ Antonius Deusinglaan 1 \\ 9713 AV Groningen \\ The Netherlands \\ *Corresponding author: w.j.quax@rug.nl
}

\section{INTRODUCTION}

The $\beta$-lactam acylases, mostly found by screening samples from natural sources, represent a unique family of heterodimeric $\mathrm{N}$-terminal nucleophile hydrolases. Interestingly, most of the strains found producing $\beta$-lactam acylases are Pseudomonas species. We have shown that these enzymes show high selectivity towards the acid side chain, but are far more promiscuous with regard to the amine moiety of the substrate. It is therefore highly unlikely that the industrially relevant deacylation of $\beta$-lactam compounds has evolved specifically in nature. Interestingly, no less than four putative acylases of the $\mathrm{N}$-terminal nucleophile family have been identified in the Pseudomonas aeruginosa $\mathrm{PAO} 1$ genome. We have investigated these four putative acylases of which one could be expressed in Escherichia coli. Interestingly, the enzyme was found to catalyse the hydrolysis of acylhomoserine-lactones as reported for Ralstonia acylase suggesting a role in quorum quenching. In a bioassay, the purified acylase was shown to degrade

Juan-Luis Ramos and Alain Filloux (eds. ), Pseudomonas, 429-449.

(C) 2007 Springer. 
acyl-HSL signal molecules with side chains ranging from 11 to 14 carbons at physiologically low concentrations confirming quorum-quenching activity. The discovery of quorum-quenching acyl-HSL acylases in the genome of $P$. aeruginosa POA1 may explain infection progression and offer new targets for anti-bacterial therapy.

\section{NTN-HYDROLASES AS $\beta$-LACTAM ACYLASES}

In search for an enzyme capable of hydrolyzing Penicillin G, in 1960 an enzyme was described which later on was used extensively for the commercial production of 6-aminopenicillanic acid (6-APA), the most important intermediate for the industrial production of semisynthetic penicillins. ${ }^{1}$ This first enzyme was named penicillin acylase (EC 3.5.1.11) and later on numerous bacterial species have been described as penicillin $\mathrm{G}$ acylase-producing strains, including E. Coli, Kluyvera citrophila and Alcaligenes faecalis. ${ }^{2,4}$ Recombinant DNA methods have been applied not only to increase the yields of commercially used penicillin G acylases, ${ }^{5}$ but also to decipher the complex processing of these enzymes. ${ }^{6}$ The penicillin $\mathrm{G}$ acylase of $E$. coli ATCC11105, a paradigm for this enzyme class, was found to be produced as a large precursor protein, which is transported into the periplasm and further processed to the mature protein constituting a small $(\alpha)$ and a large $(\beta)$ subunit. Not only this heterodimeric structure, but also the role of the N-terminal serine residue as a nucleophile for the deacylation reaction is evolutionarily preserved resulting in renaming these enzymes to $\mathrm{N}$-terminal nucleophile (NTN) hydrolases ${ }^{7}$ comprising the much larger family of $\beta$-lactam acylases.

Whereas the conversion of penicillin $G$ requires an enzyme with specificity for the aromatic phenyl acetate side chain, the processing of the second largest $\beta$-lactam fermentation product, cephalosporin-C, would require the cleavage of aminoadipyl, an aliphatic side chain, from the $\beta$-lactam nucleus. Since no enzyme capable of performing a one-step deacylation was found, ${ }^{8}$ a two enzyme-mediated reaction has been introduced to produce 7-aminocephalosporanic acid (ACA). In this process, Damino acid oxidase converts amino-adipyl into glutaryl and a glutaryl acylase performs the enzymatic deacylation to cephalosporin-C. The glutaryl acylases (EC 3.5.1.-) can be obtained from several Pseudomonas species. ${ }^{8-13}$

Interestingly, the specificity of the $\beta$-lactam acylases is mainly directed towards the acyl side chain and the enzyme is not selective to the $\beta$-lactam moiety at all. Penicillin G acylase can also hydrolyze phenylacetyl-leucine, ${ }^{4}$ and cephalosporin acylase can also hydrolyze glutarylleucine. ${ }^{14}$ This has further raised the question on the natural role of NTN- hydrolases in Gram-negative bacteria as the hydrolysis of $\beta$-lactam 